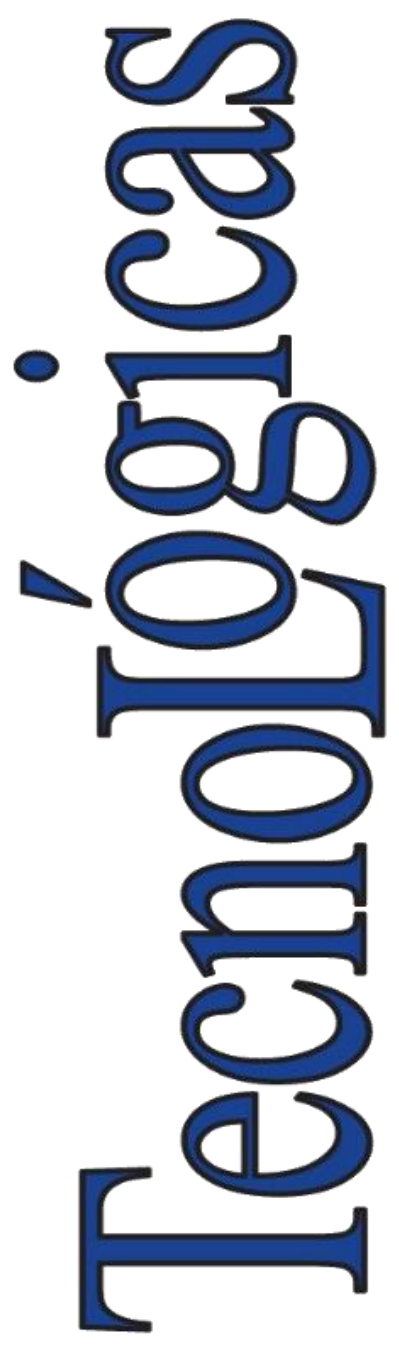

ISSN-p: 0123-7799 ISSN-e: $2256-5337$

Vol. 24, nro. 51, e1836, 2021

Recibido: 10 diciembre 2020 Aceptado: 10 mayo 2021

Disponible: 27 mayo 2021

CInstituto Tecnológico Metropolitano

Este trabajo está licenciado bajo una Licencia Internacional

Creative Commons Atribución (CC BY-NC-SA)

\section{Microencapsulación por secado por aspersión de compuestos bioactivos en diversas matrices: una revisión}

\section{Microencapsulation of Bioactive Compounds in Diverse Matrices by Spray Drying: A Literature Review}

\author{
(iD) Sara Rios-Aguirre ${ }^{1}$; \\ (D) Maritza Andrea Gil-Garzón² \\ ${ }^{1}$ Instituto Tecnológico Metropolitano, Medellín-Colombia, \\ Sararios251742@correo.itm.edu.co \\ ${ }^{2}$ Instituto Tecnológico Metropolitano, Medellín-Colombia, \\ maritzagil@itm.edu.co
}

Cómo citar / How to cite

S. Rios-Aguirre; M. A. Gil-Garzón, "Microencapsulación por secado por aspersión de compuestos bioactivos en diversas matrices: una revisión", TecnoLógicas, vol. 24, nro. 51, e1836, 2021. https://doi.org/10.22430/22565337.1836 


\section{Resumen}

La microencapsulación mediante secado por aspersión es una técnica ampliamente empleada en la protección de compuestos bioactivos, en especial sobre los polifenoles en diferentes matrices; además, es una de las técnicas más sencillas y económicas de secado, lo cual ha favorecido su transferencia tecnológica a escala industrial. Por esta razón, este artículo tiene como propósito analizar, a partir de estudios previamente reportados, los parámetros de operación empleados en la implementación de los métodos para la microencapsulación de compuestos bioactivos presentes en diversas matrices, con el fin de identificar las condiciones efectivas para futuras aplicaciones. La revisión de los estudios publicados se realizó en un período comprendido entre 2010 y 2021 en bases de datos indexadas, donde los parámetros evaluados se centraron en: las condiciones de operación, materiales encapsulantes, morfología de las microcápsulas y la eficiencia de la microencapsulación. Los resultados permitieron identificar los parámetros más críticos, entre los que sobresalen la temperatura de entrada y salida del aire, así como los materiales encapsulantes, los cuales influyen directamente en la protección de los polifenoles, que son en su mayoría termolábiles, de esta manera, se pudo constatar en los estudios revisados el alcance de una mayor eficiencia y vida útil con respecto a las propiedades funcionales, al optimizar las condiciones de operación mencionada, y se constata que los resultados reportados conllevan a la obtención de microcápsulas de matrices que pueden ser empleadas como materia prima y producto final de mayor calidad, o para estudios in vitro de liberación controlada para la industria alimentaria, farmacéutica o cosmética.

\section{Palabras clave}

Microcápsulas, secado por aspersión, capacidad antioxidante, protección de polifenoles, frutas, encapsulantes y eficiencia de encapsulación.

\section{Abstract}

Microencapsulation by spray drying is widely used to protect bioactive compounds, especially polyphenols in different matrices. It is also one of the simplest and most economical drying techniques, which has favored its technological transfer to industrial scale. Therefore, this article analyzes, based on previous studies, the operating parameters used in the implementation of methods for the microencapsulation of bioactive compounds present in various matrices, in order to identify effective conditions for future applications. This literature review covered the period between 2010 and 2021 in indexed databases, focusing on multiple parameters: operating conditions, encapsulating materials, microcapsule morphology, and microencapsulation efficiency. The results enabled us to identify the most critical parameters. Among them, air inlet and outlet temperatures stand out, as well as encapsulating materials; they directly influence the protection of polyphenols, which are mostly thermolabile. The studies reviewed here show that greater efficiency and useful life (with respect to functional properties) can be obtained by optimizing the aforementioned operating conditions. Furthermore, the results reported in this paper can be used to obtain microcapsules of matrices that can be used as a raw material, high-quality final product, or for controlled release in vitro studies in the food, pharmaceutical, or cosmetic industries.

\section{Keywords}

Microencapsulation, spray drying, antioxidant capacity, protection of polyphenols, fruits, encapsulants and encapsulation efficiency. 


\section{ABREVIATURAS}

ABTS: depleción del 2.2'-Azinobis-3-etil- benzo-tiazolina-6-ácido sulfónico.

CTF: $\quad$ contenido total de fenoles.

DE: $\quad$ equivalente dextrosa o dextrose equivalent, por sus siglas en inglés.

DPPH: depleción del óxido 2.2-difenil-1-picrilhidracilo o 2.2-diphenyl-1-picryl-hydrazylhydrate, por sus siglas en inglés.

DSC: calorímetro diferencial de barrido o differential scanning calorimeter, por sus siglas en inglés.

EM (\%): eficiencia de microencapsulación en porcentaje en masa.

FRAP: poder antioxidante reductor del hierro, por sus siglas en inglés.

GA: goma arábiga.

HPLC: cromatografía líquida de alta eficacia o high performance liquid chromatography, por sus siglas en inglés.

MD: maltodextrina.

MEB: microscopía electrónica de barrido o scanning electron microscopy (SEM, por sus siglas en inglés).

ORAC: capacidad de absorción de radicales de oxígeno u oxygen radical absorption capacity, por sus siglas en inglés.

O/W: $\quad$ aceite en agua $\mathrm{u}$ oil/water, por sus siglas en inglés.

O/W/O: aceite en agua en aceite u oil/water/oil por sus siglas en inglés.

PAS: $\quad$ principio activo superficial presente en la microcápsula (g).

PAT: $\quad$ principio activo total presente en la microcápsula (g).

PGPR: polirricinoleato de poliglicerol o polyglycerol polyricinoleate, por sus siglas en inglés.

Tg: $\quad$ temperatura de transición vítrea.

UV-Vis: ultravioleta visible.

W/O: $\quad$ agua en aceite o water/oil, por sus siglas en inglés.

W/O/W: agua en aceite en agua o water/oil/wáter, por sus siglas en inglés.

WPI: aislado de proteína de suero o Whey Protein Isolate, por sus siglas en inglés. 


\section{INTRODUCCIÓN}

Los polifenoles son compuestos de origen vegetal que se caracterizan por sus propiedades funcionales, en especial su capacidad antioxidante, los cuales se encuentran en diversas matrices, como frutas y verduras, tales como: moras [1], arándanos [2], frijol de soya [3], uvas [4], melocotón [5], guayaba [6]; cítricos, como: cascara de limón [7], mandarina, toronja [8], asaí [9]; microorganismos, como probióticos [10]; así como macrocompuestos (péptidos) [11] y microcompuestos (vitaminas y minerales) [12], entre otros. Sumado a esto, los polifenoles encontrados en una variedad de fuentes vegetales y herbales con propiedades medicinales son considerados componentes activos con capacidad de mejorar el síndromemetabólico in vitro e in vivo [13].

Hoy en día, los polifenoles representan el grupo de metabolitos secundarios más conocidos en las plantas, caracterizado por un amplio y diverso conjunto de propiedades bioactivas únicas, lo que hace que sean apreciados por sus efectos beneficiosos tanto para las plantas, como para los seres humanos. Los polifenoles son compuestos extremadamente diferentes desde el punto de vista estructural [14], que en consecuencia le permite tener diferentes funciones biológicas (antioxidantes, antiproliferantes, antinflamatorias, capacidad citotóxica, entre otras) y que, al ser incorporados en matrices alimentarias, farmacéuticas y cosméticas, le confiere propiedades funcionales que se reflejan en numerosas aplicaciones prácticas, benéficas para la salud del consumidor o conservación de las matrices.

Los polifenoles son formados mediante la ruta del ácido shikímico o poliacetato, configurándose con una estructura molecular con al menos un grupo fenol [15]. Una de las principales propiedades de los polifenoles, asociadas a su estructura química, es la capacidad que tiene sus grupos hidroxilos, los cuales actúan como centros activos, para reaccionar con radicales libres y con moléculas de peróxidos y superóxidos, propiciando la estabilidad electroquímica al neutralizar los electrones desapareados o libres, ya sea por una transferencia de electrón o hidrógeno, evitando así un deterioro celular [16], [17]. Estas reacciones se dan constantemente en las células del cuerpo humano y animal, así como estructuras vegetales, por lo cual es necesaria una protección antioxidante, la cual es proporcionada de manera natural por los polifenoles.

No obstante, es también la misma estructura química de los polifenoles que los hace sensibles a factores como: los cambios de $\mathrm{pH}$, temperatura y la presencia de oxígeno, afectando la estabilidad de sus grupos carbonilos e insaturaciones. Sumado a estos factores, los polifenoles se pueden afectar debido a la interacción con enzimas como polifenoloxidasas, peroxidasas, glicolasas y estearasas, las cuales degradan a los compuestos fenólicos en compuestos, como el ácido ascórbico. Esta falta de estabilidad de los compuestos fenólicos hace que las industrias farmacéuticas, cosmética y de alimentos se vean interesadas en la búsqueda de sistemas de protección de la capacidad antioxidante asociada a los polifenoles, si estos van a estar expuestos a condiciones ambientales o procesos que puedan disminuir su estabilidad o funcionalidad y de esta forma generar una vida útil más prolongada, facilitando su manipulación [18]. Entre las técnicas empleadas para la protección de los compuestos bioactivos y mejoramiento de su estabilidad, se encuentra la microencapsulación, la cual puede ser llevada a cabo empleando la coacervación[19], [20], secado en frío [21], secado por atomización, siendo esta última una de las de mayor aplicación en la industria de los alimentos. 


\subsection{Microencapsulación por secado por aspersión}

La microencapsulación por secado por aspersión ha sido objeto de estudio durante varias décadas y se ha encontrado en constante innovación, convirtiéndose en una de las tendencias más importantes para la industria de alimentos [22], cosmética [23], farmacéutica [24], textil [25], entre otras [26]. El secado por aspersión es una de las técnicas más antiguas de encapsulación, con un inicio que se remonta a los años 30, donde las primeras aplicaciones se enfocaron en la microencapsulación de sabores, utilizando goma acacia como material de pared o encapsulante [27].

El principio de acción del secado por aspersión utilizado para la microencapsulación consiste en: i) la alimentación a la cámara o torre de secado de una matriz, conformada por el compuesto de interés a encapsular y un material protector o encapsulante, el cual se homogeniza hasta formar una dispersión [28] o emulsión [29], dependiendo de la naturaleza del compuesto y su afinidad química con el encapsulante. Esta alimentación se realiza de manera continua y a presión mediante un sistema de boquilla o disco rotatorio [30]. ii) $\mathrm{Al}$ introducirse la matriz a la torre de secado, esta se pone en contacto con una corriente de aire caliente que puede estar en contraflujo o co-corriente y se da inicio a la eliminación rápida de la humedad de la matriz, convirtiéndose en pequeñas partículas de forma esférica, no porosas, recubiertas por una fina capa o película del encapsulante, que en su interior tienen una fase interna definida, iii) las microcápsulas formadas al salir de la cámara de secado, atraviesan un ciclón y se depositan en el colector donde pueden ser recogidas [22], [31].

La técnica de microencapsulación por secado por aspersión se caracteriza por sus bajos costos comparados con otros métodos de secado empleados [32], de allí que existan una amplia diversidad de aplicaciones, entre las que se encuentran la encapsulación de esencias [33], bebidas en polvo [34], sabores [35], probióticos [36], colorantes [37], entre otras. Como se puede observar, debido a su amplia diversidad de aplicaciones, la microencapsulación actuará como un mecanismo de protección, de acuerdo a la composición química, polaridad, solubilidad, propiedades físicas y funcionales de cada matriz, mejorando aspectos como: i) la vida útil del analito al evitar la oxidación, como en el caso de los aceites esenciales o vitaminas liposolubles; ii) prolongando la estabilidad y actividad funcional de compuestos termolábiles o altamente reactivos, como los antioxidantes; iii) facilitando la incorporación de compuestos insolubles, debido a la interacción que se facilita entre el material encapsulante y el medio o solvente en el cual se va a dispersar; iv) conservando los perfiles de aroma y sabor por un tiempo más prolongado; y por último, v) la biodisponibilidad de nutrientes al facilitar la liberación controlada en los sitios requeridos de interacción, entre otros beneficios [38], [39].

$\mathrm{Al}$ reunir las características antes descritas, asociadas al bajo costo y amplias aplicaciones en el marco de una tecnología con un principio de funcionamiento de fácil acceso para su uso y mantenimiento se convierte en una opción de gran pertinencia con un potencial importante para la transferencia tecnológica a nivel piloto e industrial, que impacta varias industrias, oportunidad que es aprovechada actualmente por países en desarrollo, en especial para aquellos con una importante riqueza en biodiversidad, ya que la microencapsulación por secado por aspersión se convierte en una opción para dar valor agregado, en especial a diversas matrices de origen vegetal con propiedades funcionales, pero que por su variedad en composición y usos, como se mencionó antes, ha sido objeto de varios estudios específicos [40].

Por lo anterior, el objetivo de este trabajo es analizar, a partir de estudios previamente reportados, los parámetros de operación empleados en la implementación de los métodos para la microencapsulación de compuestos bioactivos presentes en diversas matrices, con el fin de identificar las condiciones efectivas para futuras aplicaciones. 


\section{METOdOLOGía}

La revisión bibliográfica se llevó acabo en julio del 2020, considerando los artículos publicados entre los años 2010 y 2021, utilizando los siguientes bases de datos: Science Direct, Scopus, SciElo, American Chemical Society y Wiley. La ecuación de búsqueda empleada fue: microencapsulation AND "spray drying” AND polyphenols.

La revisión sistemática se realizó por medio de una ecuación de búsqueda utilizando los operadores booleanos AND, OR y NOT [41]. Los criterios seleccionados identifican la diferencia de concentración de polifenoles en diferentes frutas y verduras, agentes encapsulantes para el secado por aspersión, parámetros de operación en elequipo de secado, métodos de caracterización de las microcápsulas y eficiencia de microencapsulación. Todas estas publicaciones en inglés, originales y registradas en los últimos 11 años (2010-2021).

\section{RESULTADOS}

Se estudiaron 72 artículos de investigación ajustados a los términos de búsqueda utilizados, sin eliminar ningún, y se verificó que estos artículos de investigación cumplieran con los criteriosde evaluación descritos anteriormente.

De los artículos seleccionados, se encontró que los autores con más investigaciones realizadas desde el 2010 hasta el 2021 para el tema de "Microencapsulación por secado por aspersión de polifenoles", son Perego, P. el autor con más estudios realizados hasta la fecha, con cinco artículos publicados, seguido de Aliakbarian, B.; Casazza, A. A.; Estevinho, B. N.; Gimbun, J.; Pang, S. F.; y Rocha, F., con cuatro artículos publicadosy, por último, se tiene a Adamiec, J.; Ambroziak, W.; y Converti, A., los cuales presentan tres publicaciones expuestas a la fecha. Los años 2018, 2019 y 2020 representanmás del 50 \% del total de las publicaciones durante la investigación realizada, donde el país con más publicaciones fue Italia, con 13 documentos, seguido por Brasil, México, Portugal e India, con un total de 13, 11, 8, 6 y 5 publicaciones, respectivamente. De acuerdo con las afiliaciones, se tiene a la "Università degli Studi di Genova", ubicada en Italia, con seis publicaciones, seguida por la "Universidade do Porto", con cuatro publicaciones, y por último la "Universidad Veracruzana", ubicada en México, con un total de tres publicaciones. Con respecto al área temática, la cual se relaciona con el tema de estos, se destacan las siguientes:Ciencias Agropecuarias y Biológicas, con una participación del $32.2 \%$; Ingeniería Química, conel $17.5 \%$; Química, 16.8 \%; Ingeniería, $10.5 \%$, Bioquímica, Genética y Biología Molecular Biológicas, con una participación del 9.1 \% respectivamente; y Farmacología, Toxicología y Farmacia Biológicas, con el 7.0 \%.

\subsection{Microencapsulación}

Existe una variedad de métodos tanto físicos como químicos empleados para la protección de las propiedades físicas y químicas de la industria farmacéuticas, cosméticas y de alimentos, entre las cuales está la microencapsulación, que es una de las más empleadas a escala industrial [42], [43]. Debido a las matrices microencapsuladas, como vitaminas, minerales, colorantes, prebióticos, probióticos, saborizantes, antioxidantes, aceites esenciales, aromas, enzimas, drogas, e incluso fertilizantes que anteriormente se consideraban técnicamente inviables en algunos procesos productivos debido a su baja solubilidad o con una vida útil corta por su inestabilidad al estar expuestos a factores ambientales, cambios de $\mathrm{pH}$, temperatura, entre otras, ahora son posibles con la microencapsulación [44]. Dichos ingredientes están totalmente rodeados en un material de 
recubrimiento, lo que les confiere propiedades útiles de estabilidad o contrarresta las características que conllevan a la inestabilidad del ingrediente original [45]. De esta forma se obtienen microcápsulas que consisten en una membrana semipermeable, fuerte y delgada de un material polimérico, el cual rodea y a su vez contiene a la sustancia de interés, denominada centro o núcleo activo. Estas microcápsulas pueden liberar su contenido a velocidades controladas bajocondiciones específicas, a la vez que protege al compuesto encapsulado de la luz y el oxígeno, cumpliendo de esta manera su función de conservación de propiedades biológicas o fisicoquímicas.

La mayoría de las microcápsulas son esferas pequeñas con diámetros comprendido en un rango entre $0.2 \mu \mathrm{m}-5000 \mu \mathrm{m}$. El producto resultante de este proceso tecnológico recibe la denominación de "microcápsulas", "microesferas" [46], sistemas que se diferencian en morfología y estructura interna y tamaño que dependerá en gran medida del tipo de atomización y podrá variar entre $1 \mu \mathrm{m}$ y - $600 \mu \mathrm{m}$ cuando se emplea ruedas giratorias de 10 - $800 \mu \mathrm{m}$ al usar boquillas a presión de un fluido y, por último, entre $5 \mu \mathrm{m}$ - $300 \mu \mathrm{m}$ para boquillas a presión de dos fluidos [47]. Cuando las partículas poseen un tamaño inferior a 1 $\mu \mathrm{m}$, el producto resultante del proceso de microencapsulación recibe la denominación de "nanoesferas" "nano partículas" o "nanocápsulas" [35], [48], [49].

En su forma más sencilla, una microcápsula es una microesfera con pared uniforme a su alrededor, la cual se compone, por un lado, de un núcleo, relleno o fase interna que es el material que va por dentro de la microcápsula; por otro lado, está la cubierta, revestimiento, material de pared o membrana, la cual es la encargada de recubrir al material que esta por dentro de la microcápsula.

La microcápsula puede tomar diferentes formas dependiendo de varios factores como los materiales empleados, el manejo de las variables de secado, entre otras. Como se muestra en la Figura 1, existen diferentes tipos de partículas formadas a partir de la microencapsulación que pueden ser: esfera simple la cual se caracteriza por tener un recubrimiento de espesor uniforme partícula que contiene un núcleo de forma irregular; varias partículas del núcleo incrustadas en una matriz continua de material de pared; varios núcleos distintos dentro de la misma cápsula; y microcápsulas de paredes múltiples [50], [51] reportaron la encapsulación del extracto de hoja de olivo (OLE, por sus siglas en inglés) y un placebo en quitosano como material encapsulante, donde estudió la interacción de los polifenólicos presentes en OLE dentro de la matriz de polisacáridos.
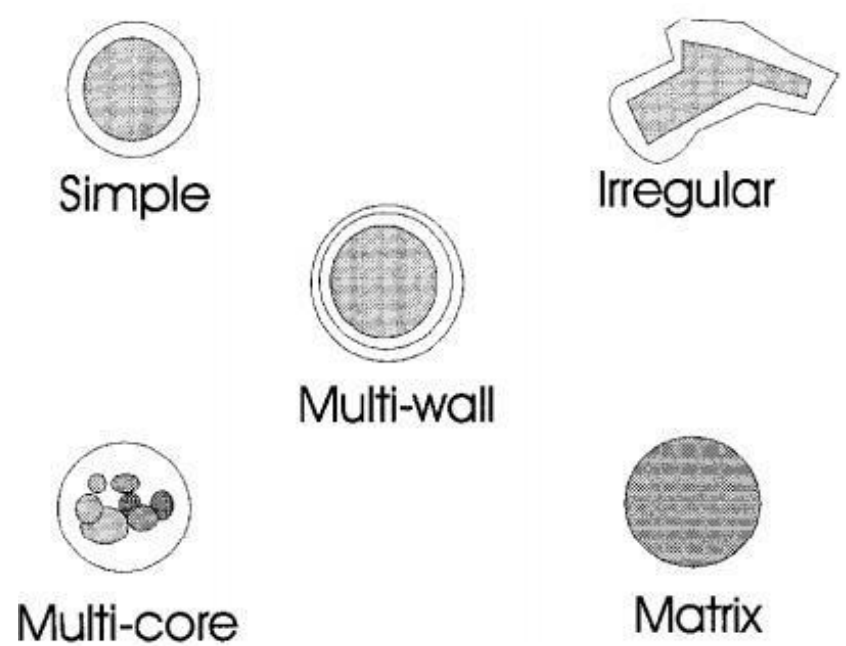

Figura 1. Tipos de partículas microencapsuladas. Fuente: [50]. 
Mediante la técnica de caracterización microscopia electrónica de barrido (MEB), se observó que las microesferas cargadas con OLE (27\%) en quitosano, revelaron una superficie perfectamente lisa, lo cual indica que existen interacciones de compuestos polifenólicos en OLE con la matriz de quitosano, con respecto a las microesferas en placebo, las cuales se mostraron con rugosidad en la superficie; además, se pudo observar espacios vacíos entre las rugosidades. Otros tipos de partículas se encontraron al microencapsular el ácido hidroxicítrico presente en el extracto de fruta de garcinia, dentro de tres matrices diferentes, que consistieron en un aislado de proteína de suero (WPI, por sus siglas en inglés), la maltodextrina (MD) y una mezcla de ambos encapsulantes (WPI + MD). Las primeras microcápsulas de WPI tenían un aspecto esférico con piel lisa y mostraron relativamente menos abolladuras y encogimiento, mientras que las cápsulas de MD y (WPI + MD) presentaban una mayor contracción y abolladuras, esto debido a la alta tasa de secado y a la rápida solidificación de la pared de la MD [52].

\subsection{Materiales encapsulantes}

Es de gran importancia la elección de un material de pared para la microencapsulación debido a que puede tener una incidencia en la eficiencia de la encapsulación y la conservación de propiedades, dándole estabilidad de las microcápsulas obtenidas.

El sistema de encapsulamiento se emplea con la finalidad de darle protección a los compuestos a encapsular de factores que pueden causar su deterioro, evitar una interacción prematura entre el material del núcleo y otros ingredientes, limitar las pérdidas volátiles y facilitar una liberación controlada o sostenida de manera dirigida de acuerdo a la aplicación que se desea realizar [27].

Un material encapsulante se define como un componente que forma un recubrimiento de capa delgada sobre una superficie. Esta capa puede ser de naturaleza orgánica, inorgánica o una combinación de ambas. Las cubiertas orgánicas, frecuentemente, presentan pigmentos, extensores y otros aditivos como catalizadores, absorbente, modificadores y antioxidantes [53]. El primer material encapsulante empleado fue la gelatina [54], pero actualmente existe una gran variedad de compuestos que responden a las diferentes matrices o compuestos que se buscan proteger por medio de la microencapsulación. En la Tabla 1 se describen características relevantes asociadas al tipo del material encapsulante como son: la cobertura específica y algunas características de interés. Estosmateriales deben de tener características ideales para ser destinados como recubrimiento en un proceso de microencapsulación en alimentos, como ha sido descrito por varios autores[21], [55]:

-Baja viscosidad a altas concentraciones.

-Facilitar la formación de película.

-Propiedades emulsificantes.

-Baja higroscopicidad, con el fin deevitar su aglomeración y facilitar su manipulación.

-Alto contenido de sólidos.

-Bajo costo.

-No poseer sabor.

- No reaccionar con el material central y ser insoluble en él.

-Soluble en la matriz donde se realizará la aplicación.

-Fácil adquisición. 
Tabla 1. Características de materiales de recubrimiento usados en la microencapsulación de alimenticios Fuente: [56].

\begin{tabular}{|c|c|c|}
\hline $\begin{array}{l}\text { Tipo de material } \\
\text { encapsulante }\end{array}$ & Cobertura específica & Características de interés \\
\hline Carbohidratos & $\begin{array}{l}\text { Almidón y derivados, maltodextrinas, jarabes } \\
\text { de maíz, sacarosa, dextrana, ciclodextrinas. }\end{array}$ & $\begin{array}{l}\text { Formador de película, muybuen } \\
\text { emulsionante. }\end{array}$ \\
\hline Gomas & $\begin{array}{l}\text { Arábiga, mezquite, guar, alginato de sodio, } \\
\text { carragenina, alginato de sodio. }\end{array}$ & $\begin{array}{l}\text { Emulsionante, formador de } \\
\text { película. }\end{array}$ \\
\hline $\begin{array}{l}\text { Tipo de material } \\
\text { encapsulante }\end{array}$ & Cobertura específica & Características de interés \\
\hline Proteínas & $\begin{array}{l}\text { Gelatina, proteína de soja, caseinatos, suero de } \\
\text { leche, zeína, gluten,caseína. }\end{array}$ & $\begin{array}{l}\text { Emulsionante, formador de } \\
\text { película. }\end{array}$ \\
\hline Lípidos & $\begin{array}{l}\text { Ceras, parafinas, grasas, ácidoesteárico, } \\
\text { triestearina, mono y diglicéridos. }\end{array}$ & $\begin{array}{l}\text { Formador de película, } \\
\text { emulsificante. }\end{array}$ \\
\hline Celulosas & $\begin{array}{l}\text { Carboximetilcelulosa, metilcelulosa, } \\
\text { etilcelulosa, nitrocelulosa, acetilcelulosa. }\end{array}$ & Formador de película. \\
\hline
\end{tabular}

La eficiencia de la microencapsulación y estabilidad de las microcápsulas durante el almacenamiento depende en la composición del material de pared. Sin embargo, varios de estos encapsulantes tienen propiedades interfaciales deficientes, por lo cual deben ser modificados químicamente o realizar una mezcla de ellos para así modificar y mejorar su actividad superficial. En adición, el conocimiento de las propiedades físicas y químicas facilitan una mayor interacción matriz: encapsulante, como las proteínas y lípidos que son moléculas anfipáticas que ofrece las propiedades emulsionantes, fisicoquímicas y funcionales necesarias para encapsular materiales básicos hidrofóbicos [57].

La goma arábiga es un polisacárido conformado por la polimerización de varios monómeros (L-arabinosa, ácido D-glucurónico y D-galactosa) [58], el cual, durante la digestión, se degrada parcialmente en el intestino grueso y es ampliamente utilizada como encapsulante,ya que presenta unas propiedades de formación de película para sabores, colores y vitaminas, además que posee buenas cualidades emulsionantes, alta solubilidad, baja viscosidad y alta eficiencia a la hora de encapsular, esto tiene relación con la estructura de la goma arábiga, ya que es un heteropolímero de azúcar altamente ramificado, que tiene una pequeña cantidad de proteína unida covalentemente a la cadena de carbohidratos, el cual actúa como un excelente agente de formación de película, facilitando la encapsulación efectiva de moléculas [59]. Sin embargo, este encapsulante es costoso y presenta problemas de suministro debido a que su producción es susceptible a las turbulencias climáticas y políticas [60]. Es por esto que varios investigadores e industrias han considerado la sustitución total o parcial de la goma arábiga por otros materiales de la pared.

Por otra parte, los carbohidratos como los almidones, los sólidos de jarabe de maíz, las maltodextrinas, las gomas como la arábiga y las proteínas como la de suero y soja se han usado generalmente como agentes encapsulantes en la industria de alimentos [61]-[63].

Estos materiales se consideran buenos agentes debido a que cumplen con las características previamente descritas, lo que promueve la productividad en el proceso de secado eliminando la etapa de hidratación previa en el proceso, y esto se ve reflejado en una alta efectividad del secado [64].

La maltodextrina es obtenida por hidrólisis enzimática o ácida del almidón y generalmente muestra una alta solubilidad en agua, sabor neutro, bajo costo, baja viscosidad a altas concentraciones y capacidad de proteger el material encapsulado de la oxidación [64], [65]. Su 
baja capacidad emulsionante puede superarse mediante el uso de almidones modificados o gomas. El almidón octenil succinato sódico es un almidón modificado químicamente que contiene un componente lipofílico que mejora su carácter anfifílico y, por lo tanto, su capacidad emulsionante [66]. Las maltodextrinas se han usado ampliamente para la microencapsulación de alimentos debido a su bajo costo, alta solubilidad y baja viscosidad a altas concentraciones. Sin embargo, la mayor desventaja de este agente de encapsulación es su falta de propiedades emulsionantes debido a su afinidad limitada por los componentes hidrófobos [67]. Por lo tanto, el uso de gomaarábiga combinada con maltodextrina puede ofrecer un buen compromiso entre costo y efectividad, como se evidencia a continuación.

En el estudio realizado por [68] propusieron una mezcla de encapsulantes, como la maltodextrina y goma arábiga en proporciones 8:2 y 6:4; cada combinación se llevó a cabo con dos equivalentes de dextrosa (4-7 y 17-20) y cada equivalente de maltodextrina también se utilizó como material encapsulante de manera individual, con el objetivo de evaluar el desempeño de estos encapsulantes sobre la estabilidad de almacenamiento en un extracto de orujo de uva microencapsulado. Esta investigación dio como resultado que la combinación de maltodextrina y goma arábiga proporciona una estabilidad sustancialmente mejorada de los polifenoles en comparación con las microcápsulas obtenidas solo con maltodextrina, siendo la mejor combinación maltodextrina MD con equivalente dextrosa 4-7 y goma arábiga ya que mostró una mejor protección que la mezcla maltodextrina MD con equivalente dextrosa 1720 y goma arábiga.

En [69] se reportan los resultados obtenidos de la microencapsulación de aceite de semilla de uva. Estos autores sugieren la adición de maltodextrina a la goma arábiga (GA) en el material de la pared en una proporción de 50:50 como una alternativa debido al aumento de los precios de la goma arábiga y su oscilación en el suministro. Ambos investigadores reportan que la encapsulación de uva con la combinación GA/MD dio como resultado una mayor actividad antioxidante de acuerdo con los métodos FRAP (poder antioxidante reductor del hierro, por sus siglas en inglés) y DPPH (depleción del óxido 2,2-difenil-1-picrilhidrazil), y un valor de peróxido más bajo que las microesferas solo con GA.

Otro material de pared es el aislado de proteína de suero (WPI, por sus siglas en inglés), el cual ayuda a la estabilidad estructural de los polvos debido a su estructura globular y su característica catiónica de $\mathrm{pH}$ ácido, lo que facilita la unión entre polisacáridos con características aniónicas,como la goma arábiga y pectina, que da como resultado un material de pared con enlaces complejos de proteína-polisacárido que favorece la microencapsulación [70]. Asimismo,WPI contiene fracciones peptídicas que pueden actuar como antioxidantes de especies reactivas de oxígeno, eliminadores de radicales libres, inhibidores de la peroxidación lipídica,quelantes de iones metálicos o una combinación de estos mecanismos, además de ser una fuente de aminoácidos [71], [72].

Un ejemplo de la aplicación de WPI fue reportada por [73] quienes investigaron sobre la microencapsulación y liberación controlada de compuestos bioactivos del orujo de uva. En este estudio utilizaron como material de pared mezclas de aislado de proteína de suero (WPI), goma arábiga (GA) y pectina. Las técnicas de microencapsulación que se utilizaron en esta investigación fueron por secado por atomización y liofilización, donde las mezclas que contenían WPI y GA en diferentes proporciones presentaban la mayor retención de compuestos fenólicos, antocianinas monoméricas totales, actividad antioxidante por ABTS (2.2-Azino-bis-3-etilbenzotiazolin-6-sulfonato) y DDPH, mientras que las pruebas de estabilidad de almacenamiento que realizaron indicaron que los polvos que contenían WPI, GA y pectina eran más estables. Los ensayos simulados de digestión gastrointestinal 
demostraron que los polvos que contenían WPI y GA como materiales de pared tenían mayores liberaciones de antocianinas en la fase intestinal.

El almidón modificado es empleado en aplicaciones alimentarias por sus propiedades de biodegradabilidad, disponibilidad y bajo costo. Este material es aplicado ampliamente para la microencapsulación de sabores que requieren de una liberacióncontrolada [74], [75]. Su composición está conformada por enlaces de cadena de amilosa y amilopectina que conllevan a una distribución helicoidal en la que cada vuelta de hélice consta de seis moléculas de glucosa[76], lo cual le permite mejorar su capacidad de interacciones químicas, en especial para el encapsulamiento; además puede ser considerado como sostenible al tener un bajo costo, abundancia y proceder de fuentes renovables, lo cual le permite estar disponible en diversas formas, dependiendo de la fuente de la materia prima [77]. Sin embargo, los almidones modificado, en especial el arrurruz (Arrowroot starch), no pueden procesarse fácilmente con calor, lo cual limita sus aplicaciones industriales [78]; es por esto que muchos investigadores lo han mezclado con otros polímeros para así mejorar sus propiedades y crear un biomaterial con aplicaciones más funcionales [79]. La mezcla del almidón con la goma arábiga tiene un gran potencial para la encapsulación, ya que la goma arábiga tiene muchas propiedades deseables como aumentar la temperatura de transición vítrea, aporta alta solubilidad, baja viscosidad y buenas propiedades emulsionantes para alimentar dispersiones [80].

En el estudio realizado por [81] se evaluó la absorción de agua y la temperatura de transición vítrea del jugo de asaí por secado por aspersión, donde usaron diferentes agentes portadores como la maltodextrina 10 DE y 20 DE, almidón de tapioca y goma arábiga, donde encontraron que los polvos obtenidos con maltodextrina $20 \mathrm{DE}$ y goma arábiga mostraron la mayor adsorción de agua, seguidos por los producidos con maltodextrina 10 DE y con almidón de tapioca, respectivamente. Con respecto a la temperatura de transición vítrea (Tg), determinada por un análisis termogravimétrico, las microcápsulas obtenidas con maltodextrina $10 \mathrm{DE}$ mostraron mayor temperatura de Tg en comparación con maltodextrina 20 DE debido a la disminución del peso molecular que influye directamente en la disminución del Tg. No obstante, aunque se esperaba un valor más alto de Tg para el almidón de tapioca al tener un peso molecular mayor que el de las maltodextrinas, se obtuvieron valores similares de Tg a causa de la baja solubilidad del almidón a temperatura ambiente, lo cual pudo producir una precipitación en la torre de secado, mientras que con la goma arábiga el comportamiento esperado, es decir, al tener un mayor peso molecular y ser soluble el jugo de asaí en este, sí se observó una Tg más alta en comparación con el polvo producido con las maltodextrinas [81].

En [82] evaluaron la liberación mediante ensayos in vitro de la microencapsulación de vitamina A mediante secado por pulverización, utilizando mezclas binarias y ternarias de goma arábiga, almidón y maltodextrina. Entre los principales hallazgos se reporta que el almidón fue el material de pared que condujo a una liberación más rápida, seguido de la goma arábiga y, finalmente, la maltodextrina, que llevó a una liberación más controlada. Con esto, al mezclar los encapsulantes, el tiempo de liberación estuvo influenciado por la acción individual de cada agente encapsulante; es decir, la presencia de almidón condujo a un tiempo más reducido y la maltodextrina contribuyó a una desaceleración de la liberación de vitamina A. La eficiencia de encapsulación osciló entre 88 \% y $98 \%$, con el valor máximo correspondiente a la mezcla ternaria.

Por lo descrito anteriormente, se evidencia la importancia del rol de los agentes encapsulantes en la acción protectora dentro de la microcápsula y cómo trae consigo otros beneficios intrínsecos, en especial en la estabilidad del producto obtenido y la liberación 
controlada en cuento la velocidad o direccionamiento del sitio específico donde se espera que se libere en compuesto bioactivo.

\subsection{Beneficios de la microencapsulación por secado por aspersión}

El principal beneficio de la microencapsulación es la protección del compuesto encapsulado por medio del recubrimiento, evitando el cambio de características sensoriales por interacciones con factores externos [42], [83].

El recubrimiento con biocompuestos, que por su estructura fisicoquímica poseen propiedades como fácil digestión, compatibilidad con la matriz alimentaria donde es incorporada la microcápsula, control de los mecanismos metabólicos a diferentes $\mathrm{pH}$ a través del tracto intestinal, permiten la liberación sostenida o controlada del principio activo, evitando así que estos compitan por los trasportadores celulares, logrando que ingrese al organismo la mayor cantidad de micronutrientes incluido en el alimento [84].

Una aplicación del beneficio antes descrito, se evidencia en el estudio realizado por [85], quienes evaluaron el efecto antimicrobiano y liberación in vitro de antocianinas de bayas y presentes en la flor de Jamaica (Hibiscus sabdariffa) microencapsuladas mediante secado por aspersión, con diferentes agentes portadores (una mezcla de goma arábiga y maltodextrina $50: 50 \mathrm{p} / \mathrm{p}$, aislado de proteína de suero (WPI) y agave fructanos). Como resultado se obtuvo que el aislado de proteína de suero fue el material de encapsulación que mayor grado de protección y retención de antocianinas logró durante las condiciones in vitro, para así alcanzar la absorción en el intestino.

En un estudio publicado en el 2019 por [86], se hablaba sobre la conservaciónde polifenoles del extracto las bayas de aronia de frutos negros (Aronia melanocarpa) y extracto del residuo de esta baya utilizando maltodextrina y leche desnatada como materiales de pared, donde las microcápsulas obtenidas con maltodextrina presentan tamaños de partículas del orden de micrones $(4.27 \mu \mathrm{m}$ para el extracto de la baya y $5.12 \mu \mathrm{m}$ para el extracto del residuo de la baya, respectivamente) en comparación con las de la leche desnatada ( $8.50 \mu \mathrm{m}$ para el extracto de la baya y $11.01 \mu \mathrm{m}$ para extracto del residuo de la baya, respectivamente). La literatura reporta que el diámetro de las partículas obtenidas mediante la microencapsulación porsecado por aspersión varía entre $10 \mu \mathrm{m}$ y $100 \mu \mathrm{m}$ [87], es por esto que los autores concluyen que las cápsulas producidas con maltodextrina pueden considerarse partículas finas, por lo que las microcápsulas proporcionan mejores propiedades tecno-funcionales que pueden ser aprovechadas para la producción de alimentos y productos farmacéuticos.

Uno de los principales beneficios de la microencapsulación es conservar las propiedades funcionales de las matrices, en este caso la capacidad antioxidante de los polifenoles, al lograr proteger estos compuestos termolábiles y sensibles a cambios de $\mathrm{pH}$ y así obtener que la adsorción en el intestino delgado. En una investigación se estudió la microencapsulación por secadopor aspersión guiado por el calorímetro diferencial de barrido (DSC, por sus siglas en inglés) de la pulpa de arazá (Eugenia stipitata), evaluando el análisis de biodisponibilidad y digestión gastrointestinal in vitro. El objetivo de estos autores [88] era preservar la bioactividad mediante el uso de microencapsulación de secado por atomización y con el uso de DSC determinar la mejor proporción de material de pared (maltodextrina o goma arábiga) y temperatura de secado $\left(100{ }^{\circ} \mathrm{C}\right.$ o $\left.120{ }^{\circ} \mathrm{C}\right)$. Estos autores proponen la proporción(1:9) de maltodextrina con una temperatura de secado de $100^{\circ} \mathrm{C}$. Con estas condiciones obtuvieron unas microcápsulas con la mejor conservación de la bioactividad después de la digestión gastrointestinal in vitro, conservando el $61 \%$ de los polifenoles totales y $101 \%$, $85 \%$ y $31 \%$ de capacidad antioxidante, según los métodos de prueba ABTS, FRAP y DPPH, respectivamente. 


\subsection{Preparación óptima de la emulsión para el método de secado por aspersión}

La microencapsulación realizada por medio de secado por aspersión inicia con la preparación de la emulsión, la cual consiste en una suspensión tipo coloide, donde un líquido en menor proporción, conocido como fase dispersa se encuentra suspendido en otro líquido inmiscible en él, considerado fase continua o dispersante que se caracteriza por estar en mayor proporción. Ambas fases, después de ser sometidos a una homogenización, forman gotas entre $0.1 \mu \mathrm{m}$ a $100 \mu \mathrm{m}$ por parte de uno de los componentes de la emulsión [89], [90].

Existen diferentes tipos de emulsiones que dependen de cómo actúa cada componente, ya sea como fase dispersa o continua. El primer tipo de emulsión es conocido como $(\mathrm{O} / \mathrm{W})$, el cual radica en una emulsión de aceite en agua que consiste en que la fase continua es el agua. El segundo tipo (W/O) consiste en gotas de agua dispersas en una fase de aceite [91]. Finalmente, existen las emulsiones múltiples de aceite en agua en aceite $(\mathrm{O} / \mathrm{W} / \mathrm{O})$ y agua en aceite en agua (W/O/W) [92]. La optimización de las emulsiones es necesarias para sumar a la efectividad de la microencapsulación en cuanto tamaño final y tipo de las cápsulas que tendrán aplicaciones de diversos sectores, como son: alimentario, cosmético, farmacia, química agrícola, industria de la pintura y polímeros, entre otros [93].

En [94] publicaron el estudio relacionado con la microencapsulación de polifenoles presentes en las hojas del romero (Rosmarinus officinalis L.) y se muestra como uno de los principales objetivos evaluar la influencia del uso de emulsiones dobles estables (W1/O/W2) durante el secado por aspersión. Como matrices utilizaron polirricinoleato de poliglicerol (PGPR) como emulsionante lipofílicoaislados de proteína de suero (WPI) (2 \% y 4 \%) como emulsionante hidrofílico y maltodextrinas (MDDE:10 y MD $_{\mathrm{DE}: 21}$ ) como portadores de recubrimiento potenciadores, donde evaluaron muestras iniciales de emulsiones dobles y muestras rehidratadas. Los resultados obtenidos muestran un efecto insignificante ( $>0.05)$ del tipo de maltodextrina usada y el contenido de proteína sobre el tamaño medio de partícula de las emulsiones dobles que contienen polifenoles de romero. Sin embargo, las emulsiones dobles de extracto fenólico de romero/aceite/agua rehidratado (RPE /O/W DE), preparado con $10 \%$ de MD y $4 \%$ de WPI, tuvo el tamaño medio de partícula máspequeño $(0.54 \mu \mathrm{m})$. Las microcápsulas rehidratadas tenían parámetros de tamaño de partícula de gota más grandes que sus doble emulsiones iniciales de $\mathrm{RPE} / \mathrm{O} / \mathrm{W}$, esto debido a la masa molecular, la cadena más larga y la menor solubilidad. Las microcápsulas rehidratadas con MDDE:10 tenían un tamaño medio de partícula significativamente mayor (hasta 8-9 veces mayor) en comparación con los DE iniciales de RPE/O/W [94].

\subsection{Parámetros de operación}

Para obtener una alta eficiencia de microencapsulación, se deben optimizar parámetros de secado por pulverización, como la temperatura de alimentación, la temperatura de entrada de aire y la temperatura de salida de aire, así como la velocidad de alimentación [95].

De acuerdo a los anterior, específicamente la temperatura de alimentación influye en la modificación de las propiedades de la emulsión, como la viscosidad, fluidez y, por lo tanto,su capacidad de pulverización homogénea, afectando la eficiencia [96]. Cabe destacar que cuando se incrementa la temperatura de alimentación, la viscosidad y el tamaño de las gotas disminuyen, pero a altas temperaturas algunos ingredientes que son sensibles al calor se pueden degradar. Por otra parte, la velocidad de alimentación que se suministra al atomizador se ajusta de acuerdo con la necesidad del nivel de secado deseado antes de llegar a la superficie de la cámara de secado. Es importante tener en cuenta el caudal y la

temperatura de entrada del aire, debido a que si esta temperatura es alta puede provocar una 
evaporación y producir grietas en la membrana, lo que conlleva a una liberación prematura y degradación del ingrediente encapsulado [97], [98]. De hecho, la temperatura de entrada del aire es directamente proporcional a la velocidad de secado de la microcápsula y al contenido final de agua. Cuando la temperatura de entrada del aire es baja, ocurre una disminución en la evaporación, lo que provoca la formación de microcápsulas de alta densidad, alto contenido de agua, poca fluidez y facilidad de aglomeración [43].

En la investigación publicada por [99], microencapsulan la uva (Vitis vinifera) mediante secado por aspersión, usando como material encapsulante la maltodextrina y goma arábiga. En este estudio evaluaron parámetros de rendimiento, higroscopicidad, contenido fenólico total y superficial, actividad antioxidante a cuatro temperaturas de entrada diferentes $\left(120^{\circ} \mathrm{C}, 140^{\circ} \mathrm{C}, 160{ }^{\circ} \mathrm{C}\right.$ y $\left.180^{\circ} \mathrm{C}\right)$, compuestos fenólicos individuales y morfología de partículas, donde como resultado obtienen que las microcápsulas con más eficiencia se presentan a una temperatura de entrada de $140{ }^{\circ} \mathrm{C}$, ya que a $120^{\circ} \mathrm{C}$ no presentaba una mayor cantidad de compuestos fenólicos en comparación con la temperatura de $140{ }^{\circ} \mathrm{C}$ y a unas temperaturas mayores comenzaron a degradar los compuestos fenólicos presentes en la uva [99].

En el estudio realizado por [100] se muestran los efectos de cinco diferentes temperaturas de entrada $\left(140{ }^{\circ} \mathrm{C}, 160{ }^{\circ} \mathrm{C}, 180^{\circ} \mathrm{C}, 200^{\circ} \mathrm{C}\right.$ y $\left.220^{\circ} \mathrm{C}\right)$ en el proceso de secado por aspersión de la sapodilla (Manilkara zapota), con base en la concentración de maltodextrina con equivalente dextrosa 10-12 en el perfil de color y el contenido total de fenoles (CTF) del polvo. Los perfiles de color y el CTF del polvo de sapodilla producido se midieron utilizando un colorímetro de Laboratorio Hunter $\left(\mathrm{L}^{*}, \mathrm{a}^{*} \mathrm{y} \mathrm{b}^{*}\right)$ y el método Folin-Ciocalteu, respectivamente. Como resultado, los autores obtuvieron que la luminosidad $\left(\mathrm{L}^{*}\right)$ de los polvos producidos con diferentes temperaturas de entrada $\left(140^{\circ} \mathrm{C}-220^{\circ} \mathrm{C}\right)$ estuvo en el rangode $92.77 \pm 0.61$ a $94.08 \pm 0.55$, donde se observó el polvo de sapodilla secado por pulverización. Cuando se aumentó la temperatura de entrada de $200{ }^{\circ} \mathrm{C}$ a $220{ }^{\circ} \mathrm{C}$, los autores advirtieron cómo el aumento de la temperatura de entrada con un grado mayor de L* conllevaron a la oxidación de algunos pigmentos presentes en la muestra. Probablemente, la reducción de la tasa de oxidación de los taninos disminuiría el polvo del color. Los colores de verde a rojo (a*) y azul a amarillo $\left(b^{*}\right)$ obtenidos con las cuatro diferentes temperaturas se encontraron en el rango de $-0.04 \pm 0.23$ a $0.15 \pm 0.14$ y $6.80 \pm 0.93$ a $8.93 \pm 1.48$, respectivamente.

Esto indica que la temperatura de entrada no afectó significativamente al perfil de los colores $\mathrm{a}^{*} \mathrm{y} \mathrm{b}^{*}$ de los polvos. Los autores indican que CTF de los polvos se vio significativamente afectado por la temperatura de entrada. Sin embargo, el aumento de la concentración de maltodextrina del $20 \%$ al $50 \%(\mathrm{p} / \mathrm{v})$ no afectó significativamente al CTF ajustado. Finalmente, los investigadores indican que los polvos de sapodilla secado por aspersión añadido con $20 \%$ o $30 \%$ (p/v) de maltodextrina y procesado a $200^{\circ} \mathrm{C}$ de temperatura de entrada, muestran un color y un CTF aceptables.

La temperatura de salida del aire es un parámetro que no puede ser controlado directamente y se dificulta establecer un rango estable de esta temperatura para una matriz, esto se debe a que depende estrictamente de las características del material a encapsular. En la literatura se ha informado que una temperatura ideal de salida está entre $50{ }^{\circ} \mathrm{C}$ y $80{ }^{\circ} \mathrm{C}$ para la microencapsulación de sabores [45].

\subsection{Métodos de caracterización y eficiencia}

La calidad de la microencapsulación se apoya en diversas técnicas como la MEB, distribución de tamaño de partícula y calorímetro diferencial de barrido [35], [88]. Así mismo, la verificación de la eficiencia se orienta a la verificación de la disponibilidad y cantidad de compuesto bioactivo encapsulado, el cual puede ser determinado mediante técnicas como la 
cromatografía líquida de alta eficacia (HPLC, por sus siglas en inglés), cromatografía de gases, espectrofotometría UV-Vis (ultravioleta visible), espectrofluorometría o mediante el establecimiento de la correlación entre el contenido total de polifenoles con su capacidad antioxidante mediante diversos métodos como: ABTS, ORAC (Capacidad de absorción de radicales de oxígeno), FRAP, DPPH, entre otros [101].

Específicamente, la técnica de microscopía electrónica de barrido se ha utilizado de manera que su análisis proporciona datos como tamaño, forma y textura de la muestra. La técnica ha evolucionado en el campo de las ciencias materiales, alimentos, la biología y la medicina [102]. Esta técnica es de gran importancia en la industria de alimentos, especialmente en la microencapsulación por secado por atomización, ya que se puede observar la morfología de las microcápsulas y determinar si el proceso de microencapsulación se dio correctamente. Como se muestra en la Figura 1, las microcápsulas pueden tomar forma simple, irregular, múltiplepared y múltiples núcleos, de las cuales la morfología definida como simple, es la requerida en microencapsulación por secado por aspersión, ya que es una estructura uniforme y lisa, la cual presenta forma ligeramente esférica con grietas y colapsos mínimos en su pared [99]. Las estructuras irregulares se dan cuando las condiciones de operación del secado por pulverización y la composición del material de revestimiento y la relación núcleo (revestimiento) no son las adecuadas.

Por otra parte, la eficiencia de la microencapsulación por secado por pulverización es relativamente alta en comparación con otros métodos, aunque no es $100 \%$ efectiva, de allí la importancia de su cuantificación [50], [91], [103]. Para la determinación de la eficiencia de microencapsulación se debe tener en cuenta la concentración del compuesto bioactivo inicial, así como es necesario cuantificar la concentración de este resultante en el núcleo de la microcápsula; finalmente será necesario determinar la cantidad del principio activo que queda en la parte externa interactuando con el material encapsulante, pero circundando el núcleo sin que se dé la protección esperada de manera efectiva al no quedar en el núcleo.

Existen varios métodos para la separación de cada componente de la microcápsula, tratamientos que se basan en la evaluación de la polaridad de sistemas de solventes que permitan interactuar de manera selectiva con el material encapsulante o compuestos bioactivo, a lo cual se le suma la aplicación de técnicas de difusión controlada o rompimiento de las cápsulas, como ultrasonido, agitación, pero también apoyados por tratamientos térmicos y variación de volúmenes de solventes que faciliten la solubilidad del material a disolver, entre otras técnicas, que dependerán de la naturaleza química de cada microencapsulación [33], [75], [87], [104].

En [33] reportan la implementación de un tratamiento para la determinación de la eficiencia de microencapsulación en una matriz lipofílica como es el aceite de café, el cual parte de la extracción con hexano para recuperar el aceite libre de la superficie que no fue encapsulado, empleando una agitación en una botella de vidrio sellada a una temperatura ambiente, seguido de una filtración, lavado que se realizó por triplicado, finalizando esta etapa con la evaporación del solvente hasta obtener peso constante y el aceite no encapsulado se calculó con base en la diferencia entre el matraz limpio inicial y el que contenía el residuo de aceite extraído. La eficiencia del aceite microencapsulado se determinó gravimétricamente mediante extracción con éter, según el método Rose-Gottlieb, reportado por Bradley et al., en 1993. Como resultado obtuvieron que la eficiencia de encapsulación varió entre 48 \% y el $82 \%$ estando influenciada positivamente con el contenido de sólidos totales, mientras que la concentración de aceite, con respecto a los sólidos totales, tuvo un efecto negativo sobre la eficiencia de encapsulación y la retención de aceite. Al igual que el aumento de la temperatura del aire de secado, también hubo una disminución tanto de la eficiencia de encapsulación como de la retención de aceite. Por esto, el proceso de microencapsulación se optimizó con un 
30 \% de sólidos totales y $15 \%$ de aceite con respecto a sólidos totales, y una temperatura de entrada de aire de $170^{\circ} \mathrm{C}$.

En complemento, la microencapsulación de champaca blanca (Michelia alba D.C) realizada por [75], logra determinar la eficiencia de microencapsulación de esta matriz de naturaleza polar. Iniciaron con la determinación del contenido superficial o libre del compuesto bioactivo, hidratando inicialmente las microcápsulas con etanol: agitando agua a $50 \mathrm{rpm}$ durante $15 \mathrm{~min}$, seguida de la evaporación al vació del etanol a $40{ }^{\circ} \mathrm{C}$ y $175 \mathrm{mbar}$ y el agua a 72 mbar. El extracto resultante fue pesado y restado de la cantidad inicial, derivando en una eficiencia de $68.91 \pm 1.5 \%$.

De los ejemplos descritos se infiere que para determinar la eficiencia de microencapsulación, se puede emplear en (1) [105].

$$
E M(\%)=\frac{P A T-P A S}{P A T} X 100
$$

Donde:

EM (\%): eficiencia de microencapsulación en porcentaje en masa.

PAT: principio activo total presente en la microcápsula (g).

PAS: principio activo superficial presente en la microcápsula (g).

En [104] investigaron sobre la microencapsulación de la curcumina (colorante natural procedente de la cúrcuma) utilizando la técnica de secado por aspersión y goma arábiga como material de pared en tres concentraciones diferentes: $10 \%, 15 \%$ y $20 \%$ (peso/volumen, w/v).

Como resultados, estos autores obtuvieron por microscopia electrónica de barrido (MEB) microcápsulas esféricas e irregulares con una superficie rugosa (sin y con curcumina). Por consiguiente, los investigadores corroboran que el encapsulante es el principal responsable de las características morfológicas de las micropartículas formadas. Todas las micropartículas presentaban una forma esférica, tenían un diámetro de alrededor de 7-9 $\mu$ m y la eficiencia de encapsulación estuvo entre el 75 \% y el $85 \%$, siendo mayor para las partículas preparadas con mayores concentraciones de agentes encapsulantes, en este caso un $20 \%$ de material de recubrimiento [104]. En los estudios de [106], el resveratrol se microencapsuló por secado por atomización utilizando goma arábiga como agente encapsulante, donde obtuvieron las micrografías por MEB de las microcápsulas con forma esférica, irregular y superficie rugosa. La eficiencia de encapsulación varió entre $82 \%$ y $87 \%$, similar a la de Bucurescu y sus coautores, siendo también mayor para las partículas preparadas con mayores concentraciones de agentes encapsulantes.

En el estudio reportado por [107], acerca de la microencapsulación de aceite de pescado mediante el secado por aspersión en presencia de goma arábiga y salvia, como material de pared, empleados de forma individual y en mezcla, conllevó a una eficiencia de encapsulación mayor en presencia de la mezcla de goma arábiga con extracto de salvia, alcanzando valores superiores a $73.21 \%$, en comparación con el $68.99 \%$ de la muestra de solo goma arábiga, lo que confirma la estabilidad estructural de la matriz de la pared (red formada por polímeros del material de la pared) con los compuestos bioactivos de interés [107].

En [108] reportan sobre el subproducto del grano de cacao basado en la extracción de agua subcrítica usando el método de secado por aspersión utilizando diferentes vehículos, como maltodextrina y proteína de suero de leche (WPI), para lo cual obtuvieron los mejores resultados al utilizar la MD como portadora con una eficacia aproximada del $74 \%$ del proceso.

Usando WPI como material portador, el contenido de fitoquímicos retenidos en la muestra encapsulada correspondió al 58.61\%. A la hora de microencapsular extracto de fruta de 
garcinia, [52] observaron resultados similares a [109] cuando los extractos de la fruta de garcinia cowa fueron secados por aspersión usando MD, WPI y su combinación.

En estudios realizados por [88], se reporta cómo la pulpa del fruto de arazá (Eugenia stipitata) microencapsulada, con maltodextrina y goma arábiga (cada una individual), presenta una mayor eficiencias de microencapsulación con MD, ya que esta conservó polifenoles totales y capacidad antioxidante en la bioactividad después de la digestión gastrointestinal in vitro, conservando el $61 \%$ de los polifenoles totales y el $101 \%$, $85 \%$ y $31 \%$ de capacidad antioxidante según los métodos de prueba ABTS, FRAP y DPPH, respectivamente.

Caracterizar las microcápsulas de acuerdo a la capacidad antioxidante final, es de gran relevancia en la justificación de microencapsular los polifenoles para su conservación, de manera que se puede garantizar la propiedad funcional atribuida a la capacidad de retardar los procesos oxidativos, consolidándose en la efectiva propiedad de antioxidante del compuesto bioactivo microencapsulado [109]. Los métodos más empleados, que previamente han sido mencionados, se pueden clasificar en diferentes grupos según su acción, como la capacidad de absorción de radicales de oxígeno (ORAC) y el potencial redox, que se mide a través del método FRAP. Existen métodos que se clasifican según la forma en que se expresen sus resultados, como la capacidad antioxidante equivalente de trolox (TEAC, por sus siglas en inglés), que incluye métodos como la reacción con el radical 2.2-difenil-1-picril hidrazilo (DPPH) y reacción con el radical 2.2'-azino-bis: 3-etilbenzotiazolin-6-sulfonato de amonio (ABTS). Ningún método refleja la capacidad antioxidante total, ya que cada uno posee mecanismos de acción diferentes sobre la forma en que actúan sobre los radicales libres responsables de los procesos oxidativos.

Estos mecanismos se caracterizan por dos acciones: transferencia de un electrón (SET) o transferencia de un protón (HAT); por esta razón es importante elucidar un perfil completo de la capacidad antioxidante asociado al contenido de polifenoles totales para determinar con mayor precisión la propiedad funcional y la efectividad del compuestos bioactivo como antioxidante sobre diferentes radicales y entender cómo va actuar según el medio o la matriz alimenticia [110].

Por lo descrito anteriormente, se evidencia que la caracterización y eficiencia de la microencapsulación refleja con mayor precisión el grado de protección que ofrece el método implementado, el cual involucra desde la caracterización inicial de la matriz a encapsular, tratamiento preliminar de la muestra, material encapsulante, hasta parámetros de operación.

Adicional a estos aspectos, se resalta la necesidad de identificar cuáles serán las variables que se tomarán como referencia para evidenciar el cumplimiento del alcance del método, que en el caso de compuestos bioactivos (como polifenoles) determinan la capacidad antioxidante y su vida útil, de manera que el método final, se convertirá en insumo para la comunidad científica e industrial al proveer el perfil o ficha técnica alcanzada, de manera que sirva como un referente para futuras aplicaciones en los sectores de alimentos, farmacia o cosmética.

\section{CONCLUSIONES}

La revisión permitió la actualización de los estudios publicados en la última década acerca de los avances generados con respecto a la microencapsulación de diferentes compuestos bioactivos, en especial polifenoles, empleando la técnica de secado por aspersión en diversas matrices como frutas, plantas, aceites de origen animal y vegetal, y semillas, entre otras.

Entre los principales hallazgos se logró evidenciar cómo el resultado de la aplicación de la técnica mejora aspectos sobre la protección de compuestos termolábiles, alargando su vida útil con respecto a sus propiedades funcionales, como la capacidad antioxidante, 
característica principal asociada al contenido de polifenoles totales, de manera que los resultados resaltados se pueden convertir en una fuente de consulta de métodos efectivos que pueden ser extrapolados a escala industrial en diferentes sectores productivos como alimentos, farmacéutica y cosmética, al igual que en estudios in vitro donde se pueda avanzar en el conocimiento de la real biodisponibilidad de los ingredientes funcionales microencapsulados.

La efectividad del método a emplear en la microencapsulación dependerá en gran medida de las propiedades y naturaleza química de la matriz de la que proviene y el tipo de compuesto bioactivo, en especial cuando se trata de polifenoles, ya que las emulsiones no se comportan de la misma manera bajo condiciones extremas de operación, donde la temperatura de entrada es un parámetro crítico que afecta de forma gradual los diferentes tipos de polifenoles, como antocianinas. Además, es fundamental en el método la elección de un material encapsulante o de una mezcla de estos, de manera que al optimizar las interacciones entre compuesto bioactivo (encapsulante, para obtener preferiblemente un tamaño de partícula y una morfología de microcápsula simple) conlleve a una eficiencia de microencapsulación mejorada y proporcional a la calidad de protección que ofrece el material de pared para los diferentes tipos de analitos de interés en diversas matrices. Por tanto, lo ideal es usar mezclas de encapsulantes en diferentes proporciones, ya que un solo material de pared no suple con todos los parámetros (formador de película, emulsificante, disponibilidad y costo) que se deben tener para formar un recubrimiento uniforme en específico. Los estudios reportan que la combinación maltodextrina con goma arábiga permite una mayor efectividad en microencapsulación.

Finalmente, tanto la caracterización como la determinación de la eficiencia de microencapsulación es necesaria para la verificación de la calidad de la aplicación del método, ya que de forma independiente no es suficiente para hacer una correcta trazabilidad y proyección de la efectividad del producto final microencapsulado, en especial en procesos de liberación controlada.

\section{AGRADECIMIENTOS}

Los autores agradecen a la convocatoria Jóvenes Investigadores e Innovadores, para grupos de investigación del ITM-2020, por su financiamiento; asimismo, al proyecto P20245 "Evaluación de la capacidad antioxidante y función biológica de un extracto y jugo obtenido a partir de la uva Isabella (Vitis labrusca) mediante la caracterización del perfil quimiométrico y de la actividad citotóxica y antitumoral: una opción para ampliar la aplicación de un ingrediente funcional" de la convocatoria interna ITM-2019 en convenio con la Corporación Universitaria Lasallista.

\section{CONFLICTOS DE INTERÉS DE LOS AUTORES}

No existe entre los autores conflictos de intereses de tipo financiero, profesional o personal, que hubieran podido afectar de manera directa en el desarrollo de la presente revisión. 


\section{CONTRIBUCIÓN DE LOS AUTORES}

Tanto la concepción como la redacción y análisis de la información fue realizada de manera conjunta por los autores.

El autor de correspondencia, además de contribuir de manera relevante en la redacción e interpretación de los resultados, aportó en la orientación de la temática y diseño metodológico de búsqueda.

Los aportes específicos consistieron en:

Sara Ríos-Aguirre, quien participó en la compilación de los artículos y en la revisión y análisis de estos.

Maritza Andrea Gil-Garzón, quien participó en la concesión del alcance del artículo, así como en el análisis y las conclusiones.

\section{REFERENCIAS}

[1] T. C. Buttow Rigolon; F. A. Ribeiro de Barros; É. N. Rufino Vieira; P. C. Stringheta, "Prediction of total phenolics, anthocyanins and antioxidant capacity of blackberry (Rubus sp.), blueberry (Vaccinium sp.) and jaboticaba (Plinia cauliflora (Mart.) Kausel) skin using colorimetric parameters," Food Sci. Technol., vol. 40, no. suppl 2, pp. 620-625, May. 2020. https://doi.org/10.1590/fst.34219

[2] Y. Wei et al., "Exploring the biochemical properties of three polyphenol oxidases from blueberry (Vaccinium corymbosum L.)," Food Chem., vol. 344, May 2021. https://doi.org/10.1016/j.foodchem.2020.128678

[3] I. Cabezudo; M. R. Meini; C. C. Di Ponte; N. Melnichuk; C. E. Boschetti; D. Romanini, "Soybean (Glycine max) hull valorization through the extraction of polyphenols by green alternative methods," Food Chem., vol. 338, Feb. 2021. https://doi.org/10.1016/j.foodchem.2020.128131

[4] E. Elejalde; M. C. Villarán; R. M. Alonso, "Grape polyphenols supplementation for exercise-induced oxidative stress," Journal of the International Society of Sports Nutrition, vol. 18, no. 3, Jan. 2021. https://doi.org/10.1186/s12970-020-00395-0

[5] C. Guo et al., "Immunomodulation effects of polyphenols from thinned peach treated by different drying methods on RAW264.7 cells through the NF-kB and Nrf2 pathways," Food Chem., vol. 340, Mar. 2021. https://doi.org/10.1016/j.foodchem.2020.127931

[6] H. Shabbir et al., "In vivo screening and antidiabetic potential of polyphenol extracts from guava pulp, seeds and leaves," Animals, vol. 10, no. 9, pp. 1-14, Sep. 2020. https://doi.org/10.3390/ani10091714

[7] R. Nawaz; N. Safdar; A. Ainee; S. Jabbar, "Development and storage stability studies of functional fruit drink supplemented with polyphenols extracted from lemon peels," J. Food Process. Preserv., vol. 45, no. 3, Jan. 2021. https://doi.org/10.1111/jfpp.15268

[8] E. S. Ordoñez-Gómez; D. Reátegui-Díaz; J. E. Villanueva-Tiburcio, "Total polyphenols and antioxidant capacity of peel and leaves in twelve citrus," Sci. Agropecu., vol. 9, no. 1, pp. 123-131, Mar. 2018. URL

[9] B. A. Rojano; I. C. Zapata Vahos; A. F. Alzate Arbeláez; A. J. Mosquera Martínez; F. Bernardo Cortés Correa; L. Gamboa Carvajal, "Polifenoles y Actividad Antioxidante del Fruto Liofilizado de Palma Naidi (Açai Colombiano) (Euterpe oleracea Mart)" Rev. Fac. Nac. Agron., vol. 64, no. 2, pp. 6213-6220, 2011. URL

[10] S. Rodríguez Barona; L. M. Montes; D. D. J. Ramírez, "Microencapsulación De Probióticos Mediante Secado Por Aspersión En Presencia De Prebióticos,” Vitae, vol. 19, no. 1, pp. S186-S188, Jan. 2012. URL

[11] K. Sarabandi; P. Gharehbeglou; S. M. Jafari, "Spray-drying encapsulation of protein hydrolysates and bioactive peptides: Opportunities and challenges," Dry. Technol., vol. 38, no. 5-6, pp. 577-595, Nov. 2019. https://doi.org/10.1080/07373937.2019.1689399

[12] S. R. Bajaj; S. J. Marathe; R. S. Singhal, "Co-encapsulation of vitamins B12 and D3 using spray drying: Wall material optimization, product characterization, and release kinetics," Food Chem., vol. 335, p. 127642, Jan. 2021. https://doi.org/10.1016/j.foodchem.2020.127642

[13] L. Chen; H. Cao; J. Xiao, "Polyphenols: Absorption, bioavailability, and metabolomics", in Polyphenols: Properties, Recovery, and Applications, Elsevier, 2018, pp. 45-67. https://doi.org/10.1016/B978-0-12813572-3.00002-6

[14] A. Belščak-Cvitanović; K. Durgo; A. Huđek; V. Bačun-Družina; D. Komes, "Overview of polyphenols and their properties," in Polyphenols: Properties, Recovery, and Applications, Elsevier, 2018, pp. 3-44. https://doi.org/10.1016/B978-0-12-813572-3.00001-4 
[15] M. Quiñones; M. Miguel; A. Aleixandre, "Los polifenoles, compuestos de origen natural con efectos saludables sobre el sistema cardiovascular," Nutr. hosp, vol. 27, no. 1, pp. 76-89, 2012. https://doi.org/10.3305/nh.2012.27.1.5418

[16] D. A. Martín Gordo, "Los Compuestos Fenólicos, Un Acercamiento A Su Biosíntesis, Síntesis Y Actividad Biológica," Rev. Investig. Agrar. y Ambient., vol. 9, no. 1, pp. 81-104, Feb. 2018. https://doi.org/10.22490/21456453.1968

[17] M. Avello; M. Suwalsky, "Radicales libres, antioxidantes naturales y mecanismos de protección," Atenea (Concepción), no. 494, pp. 161-172, 2006. http://dx.doi.org/10.4067/S0718-04622006000200010

[18] S. Ray; U. Raychaudhuri; R. Chakraborty, "An overview of encapsulation of active compounds used in food products by drying technology," Food Biosci., vol. 13, pp. 76-83, Mar. 2016. https://doi.org/10.1016/j.fbio.2015.12.009

[19] N. Kanha; J. M. Regenstein; S. Surawang; P. Pitchakarn; T. Laokuldilok, "Properties and kinetics of the in vitro release of anthocyanin-rich microcapsules produced through spray and freeze-drying complex coacervated double emulsions," Food Chem., vol. 340, Mar. 2021. https://doi.org/10.1016/j.foodchem.2020.127950

[20] R. Tan et al., "Preparation of Green Coffee Oil Microcapsules by Complex Coacervation Method and Its Physicochemical Properties," Shipin Kexue/Food Sci., vol. 41, no. 23, pp. 144-152, Dec. 2020. https://doi.org/10.7506/spkx1002-6630-20191128-273

[21] R. A. Parra Huertas, "Revisión: Microencapsulación de Alimentos,” Rev. Fac. Nac. Agron., vol. 63, no. 2, pp. 5669-5684, Jun.2010. https://www.redalyc.org/pdf/1799/179918602020.pdf

[22] S. Risch et al., Encapsulation and controlled release of food ingredients. Washington, DC, EEUU: ACS Symposium Series; American Chemical Society, 1995, p. 214. https://doi.org/10.1021/bk-1995-0590.fw001

[23] M. R. Barroso et al., "Exploring the antioxidant potential of Helichrysum stoechas (L.) Moench phenolic compounds for cosmetic applications: Chemical characterization, microencapsulation and incorporation into a moisturizer"," Ind. Crops Prod., vol. 53, pp. 330-336, Feb. 2014. https://doi.org/10.1016/j.indcrop.2014.01.004

[24] D. Krajišnik; B. Čalija; N. Cekić, "Polymeric Microparticles and Inorganic Micro/Nanoparticulate Drug Carriers: An Overview and Pharmaceutical Application," in Microsized and Nanosized Carriers for Nonsteroidal Anti-Inflammatory Drugs, Elsevier, 2017, pp. 31-67. https://doi.org/10.1016/B978-0-12$\underline{804017-1.00002-9}$

[25] R. Badulescu; V. Vivod; D. Jausovec; B. Voncina, "Treatment of Cotton Fabrics with Ethyl Cellulose Microcapsules," in Medical and Healthcare Textiles, Woodhead publishing, 2010, pp. $226-235$. https://doi.org/10.1533/9780857090348.226

[26] S. Gouin, "Microencapsulation: Industrial appraisal of existing technologies and trends," Trends in Food Science and Technology, vol. 15, no. 7-8, pp. 330-347. Aug. 2004. https://doi.org/10.1016/j.tifs.2003.10.005

[27] F. Shahidi; X. Qing Han, "Encapsulation of Food Ingredients," Crit. Rev. Food Sci. Nutr., vol. 33, no. 6, pp. 501-547, Jan. 1993. https://doi.org/10.1080/10408399309527645

[28] M. L. Bruschi, "Drug delivery systems," in Strategies to Modify the Drug Release from Pharmaceutical Systems, Woodhead Publishing, 2015, pp. 87-194. https://doi.org/10.1016/B978-0-08-100092-2.00006-0

[29] M. Jelvehgari; S. Hassan Montazam, "Comparison of Microencapsulation by Emulsion-Solvent Extraction/ Evaporation Technique Using Derivatives Cellulose and Acrylate-Methacrylate Copolymer as Carriers," Jundishapur J. Nat. Pharm. Prod., vol. 7, no. 4, pp. 144-152, Oct. 2012. https://doi.org/10.5812/jinpp.3986

[30] A. Ye; S. G. Anema; H. Singh, "Behaviour of homogenized fat globules during the spray drying of whole milk," Int. Dairy J., vol. 17, no. 4, pp. 374-382, Apr. 2007. https://doi.org/10.1016/j.idairyj.2006.04.007

[31] K.-Y. Show; Y.-G. Yan; D.-J. Lee, "Chapter 7 - Algal biomass harvesting and drying," in Biofuels from Algae, Elsevier, 2019, pp. 135-166. https://doi.org/10.1016/B978-0-444-64192-2.00007-X

[32] T. Moreno et al., "Spray Drying Formulation of Polyphenols-Rich Grape Marc Extract: Evaluation of Operating Conditions and Different Natural Carriers," Food Bioprocess Technol., vol. 9, no. 12, pp. 20462058, Dec. 2016. https://doi.org/10.1007/s11947-016-1792-0

[33] E. C. Frascareli; V. M. Silva; R. V. Tonon; M. D. Hubinger, "Effect of process conditions on the microencapsulation of coffee oil by spray drying," Food Bioprod. Process., vol. 90, no. 3, pp. 413-424, Jul. 2012. https://doi.org/10.1016/j.fbp.2011.12.002

[34] G. A. Reineccius, "The Spray Drying of Food Flavors," Dry. Technol., vol. 22, no. 6, pp. 1289-1324, Jun. 2004. https://doi.org/10.1081/DRT-120038731

[35] J. Shaikh; R. Bhosale; R. Singhal, "Microencapsulation of black pepper oleoresin," Food Chem., vol. 94, no. 1, pp. 105-110, Jan. 2006. https://doi.org/10.1016/j.foodchem.2004.10.056

[36] O. D . López Hernandez; J. K. Lozada López, "Microencapsulación del probiótico Saccharomyces cerevisiae var. boulardii mediante secado por aspersión con polímeros Eudragitß,” 2021. https://repositorio.uta.edu.ec/handle/123456789/32109

[37] D. B. Rodriguez-Amaya, "Natural food pigments and colorants," Current Opinion in Food Science, vol. 7. 
pp. 20-26, Feb. 2016 https://doi.org/10.1016/j.cofs.2015.08.004

[38] V. B. de Souza; M. Thomazini; J. C. D. Carvalho Balieiro; C. S. Fávaro-Trindade, "Effect of spray drying on the physicochemical properties and color stability of the powdered pigment obtained from vinification byproducts of the Bordo grape (Vitis labrusca)," Food Bioprod. Process., vol. 93, pp. 39-50, Jan. 2015. https://doi.org/10.1016/j.fbp.2013.11.001

[39] T. Moreno; M. J. Cocero; S. Rodríguez-Rojo, "Storage stability and simulated gastrointestinal release of spray dried grape marc phenolics," Food Bioprod. Process., vol. 112, pp. 96-107, Nov. 2018. https://doi.org/10.1016/j.fbp.2018.08.011

[40] I. Khalifa; M. Li; T. Mamet; C. Li, "Maltodextrin or gum Arabic with whey proteins as wall-material blends increased the stability and physiochemical characteristics of mulberry microparticles," Food Biosci., vol. 31, Oct. 2019. https://doi.org/10.1016/j.fbio.2019.100445

[41] J. A. Cardona Arias; L. F. Higuita Gutiérrez; L. A. Ríos Osorio, Revisiones sistemáticas de la literatura científica: La investigación teórica como principio para el desarrollo de la ciencia básica y aplicada. Universidad Cooperativa de Colombia, 2016. http://dx.doi.org/10.16925/9789587600377

[42] M. Cano-Chauca; P. C. Stringheta; A. M. Ramos; J. Cal-Vidal, "Effect of the carriers on the microstructure of mango powder obtained by spray drying and its functional characterization," Innov. Food Sci. Emerg. Technol., vol. 6, no. 4, pp. 420-428, Dec. 2005. https://doi.org/10.1016/j.ifset.2005.05.003

[43] M. C. Otálora; J. G. Carriazo; L. Iturriaga; M. A. Nazareno; C. Osorio, "Microencapsulation of betalains obtained from cactus fruit (Opuntia ficus-indica) by spray drying using cactus cladode mucilage and maltodextrin as encapsulating agents," Food Chem., vol. 187, pp. 174-181, Nov. 2015. https://doi.org/10.1016/j.foodchem.2015.04.090

[44] H. Castañeta; R. Gemio; W. Yapu; J. Nogales, "Microencapsulación, un método para la conservación de propiedades fisicoquímicas y biológicas de sustancias químicas," Rev. Boliv. Química, vol. 28, no. 2, pp. 135140, 2011.https://www.redalyc.org/articulo.oa?id=426339676015

[45] A. Gharsallaoui; G. Roudaut; O. Chambin; A. Voilley; R. Saurel, "Applications of spray-drying in microencapsulation of food ingredients: An overview," Food Res. Int., vol. 40, no. 9, pp. 1107-1121, Nov. 2007. https://doi.org/10.1016/j.foodres.2007.07.004

[46] F. Fu; L. Hu, "Temperature sensitive colour-changed composites," in Advanced High Strength Natural Fibre Composites in Construction, Woodhead Publishing., 2017, pp. 405-423 http://dx.doi.org/10.1016/B978-0-08100411-1.00015-7

[47] I. Filková; A. S. Mujumdar, "Industrial spay Drying systemns", in Handbook of Industrial Drying, Second Edition, Revised and Expanded. 1995. URL

[48] O. Anselmino; E. Gilg, "Emulsionen" in Kommentar zum Deutschen Arzneibuch 6. Ausgabe 1926, Berlin, Springer 1928, pp. 532-535. https://doi.org/10.1007/978-3-642-90745-6_200

[49] M. Lozano Berna, "Obtención de microencapsulados funcionales de zumo de opuntia stricta mediante secado por atomización,” Ing. Técnica Ind. Espec. en Química Insdustrial. Univ. Politécnica Cart., pp. 169, 2009. URL

[50] B. F. Gibbs; S. Kermasha; I. Alli; C. N. Mulligan, "Encapsulation in the food industry: a review," Int. J. Food Sci. Nutr., vol. 50, no. 3, pp. 213-224, Jan. 1999. https://doi.org/10.1080/096374899101256

[51] S. L. Kosaraju; L. D'ath; A. Lawrence, "Preparation and characterisation of chitosan microspheres for antioxidant delivery," Carbohydr. Polym., vol. 64, no. 2, pp. 163-167, May 2006. https://doi.org/10.1016/j.carbpol.2005.11.027

[52] P. Natarajan Ezhilarasi; D. Indrani; B. Sankar Jena; C. Anandharamakrishnan, "Microencapsulation of Garcinia fruit extract by spray drying and its effect on bread quality," J. Sci. Food Agric., vol. 94, no. 6, pp. 1116-1123, Apr. 2014. https://doi.org/10.1002/jsfa.6378

[53] V. M. Dávalos, "Nanoencapsulación de Riboflavina en matrices poliméricas biodegradables empleando la técnica de secado por aspersión," Instituto Politécnico -Nacional, Centro de investigación en ciencia aplicada y tecnologia unidad Legaria cicata IPN, México D.F, 2014. URL

[54] G. O. Fanger, "Microencapsulation: A Brief History and Introduction," in Microencapsulation, Boston, MA: Springer US, 1974, pp. 1-20. https://doi.org/10.1007/978-1-4684-0739-6_1

[55] J. Luna Guevara; J. López Fuentes; O. Jiménez Gonzalez; L. Luna Guevara "Microencapsulación de algunos compuestos bioactivos mediante secado por aspersión”, Rev. Iberoam. las Ciencias Biológicas y Agropecu., vol. 5, no. 10, pp. 11, Aug 2016. https://doi.org/10.23913/ciba.v5i10.56

[56] E. N. Reyna; G. M. Álvarez; A. Iliná; J. L. Martinez Hernández, "Microencapsulación de componentes bioactivos," Investig. y Ciencia, Univ. Autónoma Aguascalientes, vol. 23, no. 64, pp. 2-8, Spe. 2015. URL

[57] J. Torres García; S. Dura Agüero, "Fosfolípidos: Propiedades y efectos sobre la salud," Nutr. Hosp., vol. 31, no. 1, pp. 76-83, 2015. http://dx.doi.org/10.3305/nh.2015.31.1.7961

[58] C. V. Córdoba, "Las plantas en la poesía de góngora (en el tricentésimo nonagésimo aniversario de su muerte)," Madrid, 2018. URL

[59] V. M. Burin; P. N. Rossa; N. E. Ferreira-Lima; M. C. R. Hillmann; M. T. Boirdignon-Luiz, "Anthocyanins: 
optimisation of extraction from Cabernet Sauvignon grapes, microcapsulation and stability in soft drink," Int. J. Food Sci. Technol., vol. 46, no. 1, pp. 186-193, Jan. 2011. https://doi.org/10.1111/j.13652621.2010.02486.x

[60] A. M. Kalušević et al., "Effects of different carrier materials on physicochemical properties of microencapsulated grape skin extract,” J. Food Sci. Technol., vol. 54, no. 11, pp. 3411-3420, Oct. 2017. https://doi.org/10.1007/s13197-017-2790-6

[61] P. Labuschagne, "Impact of wall material physicochemical characteristics on the stability of encapsulated phytochemicals: A review," Food Res. Int., vol. 107, pp. 227-247, May. 2018. https://doi.org/10.1016/j.foodres.2018.02.026

[62] M. Rostami; M. Yousefi; A. Khezerlou; M. Aman Mohammadi; S. M. Jafari, "Application of different biopolymers for nanoencapsulation of antioxidants via electrohydrodynamic processes," Food Hydrocoll., vol. 97, p. 105170, Dec. 2019, https://doi.org/10.1016/j.foodhyd.2019.06.015

[63] M. M. Kenyon, "Modified Starch, Maltodextrin, and Corn Syrup Solids as Wall Materials for Food Encapsulation," Encapsulation and controlled release of food ingredients, 1995, pp. 42-50. https://doi.org/10.1021/bk-1995-0590.ch004

[64] Z. A. Özbek; P. G. Ergönül, "Optimisation of wall material composition of freeze-dried pumpkin seed oil microcapsules: Interaction effects of whey protein, maltodextrin, and gum Arabic by D-optimal mixture design approach," Food Hydrocoll., vol. 107, p. 105909 , Oct. 2020. https://doi.org/10.1016/j.foodhyd.2020.105909

[65] M. L. Martínez et al., "Oxidative stability of walnut (Juglans regia L.) and chia (Salvia hispanica L.) oils microencapsulated by spray drying," Powder Technol., vol. 270, no. Part A, pp. 271-277, Jan. 2015. https://doi.org/10.1016/j.powtec.2014.10.031

[66] E. C. Quirino Lacerda; V. M. De Araujo Calado; M. Monteiro; P. V. Finotelli; A. Guedes Torres; D. Perrone, "Starch, inulin and maltodextrin as encapsulating agents affect the quality and stability of jussara pulp microparticles," Carbohydr. Polym., vol. 151, pp. 500-510, Oct. 2016. https://doi.org/10.1016/j.carbpol.2016.05.093

[67] T. Dey, "Book Review," J. Dispers. Sci. Technol., vol. 33, no. 6, pp. 928-932, May. 2012. https://doi.org/10.1080/01932691.2011.584482

[68] A. Tolun; N. Artik; Z. Altintas, "Effect of different microencapsulating materials and relative humidities on storage stability of microencapsulated grape pomace extract," Food Chem., vol. 302, p. 125347, Jan. 2020. https://doi.org/10.1016/j.foodchem.2019.125347

[69] L. S. Kuck; C. Pelayo Zapata Noreña, "Microencapsulation of grape (Vitis labrusca var. Bordo) skin phenolic extract using gum Arabic, polydextrose, and partially hydrolyzed guar gum as encapsulating agents," Food Chem., vol. 194, pp. 569-576, Mar. 2016. https://doi.org/10.1016/j.foodchem.2015.08.066

[70] C. Chung; T. Rojanasasithara; W. Mutilangi; D. J. McClements, "Enhanced stability of anthocyanin-based color in model beverage systems through whey protein isolate complexation," Food Res. Int., vol. 76, pp. 761-768, Oct. 2015. https://doi.org/10.1016/j.foodres.2015.07.003

[71] F. P. Flores; R. K. Singh; W. L. Kerr; R. B. Pegg; F. Kong, "Total phenolics content and antioxidant capacities of microencapsulated blueberry anthocyanins during in vitro digestion," Food Chem., vol. 153, pp. 272-278, Jun. 2014. https://doi.org/10.1016/j.foodchem.2013.12.063

[72] A. Brandelli; D. J. Daroit; A. P. Folmer Corrêa, "Whey as a source of peptides with remarkable biological activities," Food Res. Int., vol. 73, pp. 149-161, Jul. 2015. https://doi.org/10.1016/j.foodres.2015.01.016

[73] C. Brown da Rocha; C. Pelayo Zapata Noreña, "Microencapsulation and controlled release of bioactive compounds from grape pomace," Dry. Technol., pp. 1-15, Mar. 2020. https://doi.org/10.1080/07373937.2020.1741004

[74] I. F. Nata; K.-J. Chen; C.-K. Lee, "Facile microencapsulation of curcumin in acetylated starch microparticles," J. Microencapsul., vol. 31, no. 4, pp. 344-349, Jun. 2014. https://doi.org/10.3109/02652048.2013.858789

[75] R. Samakradhamrongthai; P. Thakeow; P. Kopermsub; N. Utama-ang, "Microencapsulation of white champaca (Michelia alba D.C.) extract using octenyl succinic anhydride (OSA) starch for controlled release aroma," J. Microencapsul., vol. 33, no. 8, pp. 773-784, Nov. 2016. https://doi.org/10.1080/02652048.2016.1264493

[76] S. Badui, Química de los alimentos. 2006. URL

[77] T. Sartori; F. C. Menegalli, "Development and characterization of unripe banana starch films incorporated with solid lipid microparticles containing ascorbic acid," Food Hydrocoll., vol. 55, pp. 210-219, Apr. 2016. https://doi.org/10.1016/j.foodhyd.2015.11.018

[78] G. Ferreira Nogueira; L. G. Pereira Martin; F. Matta Fakhouri; R. Augustus de Oliveira, "Microencapsulation of blackberry pulp with arrowroot starch and gum arabic mixture by spray drying," $J$. Microencapsul., vol. 35, no. 5, pp. 482-493, Oct. 2018. https://doi.org/10.1080/02652048.2018.1538264

[79] C.-S. Wu; H.-T. Liao, "Interface design and reinforced features of arrowroot (Maranta arundinacea) 
starch/polyester-based membranes: Preparation, antioxidant activity, and cytocompatibility," Mater. Sci. Eng. C, vol. 70, no. 1, pp. 54-61, Jan. 2017. https://doi.org/10.1016/j.msec.2016.08.067

[80] M. J. Ramírez; G. I. Giraldo; C. E. Orrego, "Modeling and stability of polyphenol in spray-dried and freezedried fruit encapsulates," Powder Technol., vol. 277, pp. 89-96, Jun. 2015. https://doi.org/10.1016/j.powtec.2015.02.060

[81] R. V. Tonon; A. F. Baroni; C. Brabet; O. Gibert; D. Pallet; M. D. Hubinger, "Water sorption and glass transition temperature of spray dried açai (Euterpe oleracea Mart.) juice," J. Food Eng., vol. 94, no. 3-4, pp. 215-221, Oct. 2009. https://doi.org/10.1016/j.jfoodeng.2009.03.009

[82] A. M. Ribeiro; M. Shahgol; B. N. Estevinho; F. Rocha, "Microencapsulation of Vitamin A by spray-drying, using binary and ternary blends of gum arabic, starch and maltodextrin," Food Hydrocoll., vol. 108, p. 106029, Nov. 2020. https://doi.org/10.1016/j.foodhyd.2020.106029

[83] Y. Herrera Ardila, "Microencapsulación de compuestos con poder antioxidante extraídos a partir de semillas sin fermentar de Theobroma cacao y Theobroma grandiflorum," (Tesis Maestria) Universidad Nacional de Colombia, Facultad de Ciencias, Maestría en Ciencia y Tecnología de Alimentos, Bogotá D.C. 2013. URL

[84] K. Goud; H. Desai; H. Jin Park, "Recent Developments in Microencapsulation of Food Ingredients," Dry. Technol., vol. 23, no. 7, pp. 1361-1394, Jul. 2005. https://doi.org/10.1081/DRT-200063478

[85] V. S. Farias-Cervantes; A. Chávez-Rodríguez; P. A. García-Salcedo; P. M. García-López; J. Casas-Solís; I. Andrade-González, "Antimicrobial effect and in vitro release of anthocyanins from berries and Roselle obtained via microencapsulation by spray drying," J. Food Process. Preserv., vol. 42, no. 10, p. e13713, Oct. 2018. https://doi.org/10.1111/jfpp.13713

[86] N. Ćujić-Nikolić et al., "Chokeberry polyphenols preservation using spray drying: effect of encapsulation using maltodextrin and skimmed milk on their recovery following in vitro digestion," J. Microencapsul., vol. 36, no. 8, pp. 693-703, Nov. 2019. https://doi.org/10.1080/02652048.2019.1667448

[87] N. V. Naga Jyothi; P. Muthu Prasanna; S. Narayan Sakarkar; K. Surya Prabha; P. Seetha Ramaiah; G. Y. Srawan, "Microencapsulation techniques, factors influencing encapsulation efficiency," J. Microencapsul., vol. 27, no. 3, pp. 187-197, May 2010. https://doi.org/10.3109/02652040903131301

[88] M. Soria Iturri; C. M. Barros Calado; C. Prentice, "Microparticles of Eugenia stipitata pulp obtained by spray-drying guided by DSC: An analysis of bioactivity and in vitro gastrointestinal digestion," Food Chem., vol. 334, pp. 127557, Jan. 2021. https://doi.org/10.1016/j.foodchem.2020.127557

[89] D. J. McClements, Food Emulsions. CRC Press, 2015. https://doi.org/10.1201/b18868

[90] C. Anandharamakrishnan; S. Padma Ishwarya, Spray Drying Techniques for Food Ingredient Encapsulation. Chichester, UK: John Wiley \& Sons, Ltd, 2015. https://doi.org/10.1002/9781118863985.ch11

[91] C. Anandharamakrishnan; S. P. Ishwarya, Spray Drying Techniques for Food Ingredient Encapsulation, Modeling approach for spray drying and encapsulation applications Chichester, UK: John Wiley \& Sons, Ltd, 2015. https://doi.org/10.1002/9781118863985.ch11

[92] P. Becher, "Encyclopedia of Emulsion Technology - Volume I - Basic Theory. New York - Basel, 1983.

[93] J. Muñoz; M. D. C. Alfaro; I. Zapata, "Progress in emulsion formulation," Grasas y Aceites, vol. 58, no. 1, pp. 64-73, Mar. 2007. https://doi.org/10.3989/gya.2007.v58.i1.10

[94] A. Bušić et al., "The Potential of Combined Emulsification and Spray Drying Techniques for Encapsulation of Polyphenols from Rosemary (Rosmarinus officinalis L.) Leaves,” Food Technol. Biotechnol., vol. 56, no. 4, pp. 494-505, 2018. https://doi.org/10.17113/ftb.56.04.18.5680

[95] Z. Liu; J. Hong Zhou; Y. Long Zeng; X. Long Ouyang, "The enhancement and encapsulation of Agaricus bisporus flavor," J. Food Eng., vol. 65, no. 3, pp. 391-396, Dec. 2004. https://doi.org/10.1016/j.jfoodeng.2004.01.038

[96] B. E. Esquivel González; L. A Ochoa Martínez; O.M. Rutiaga Quiñonez, "Microencapsulación mediante secado por aspersión de compuestos bioactivos," Rev. Iberoam. Tecnol. Postcosecha, vol. 16, no. 2, pp. 180192, Jun. 2015. URL

[97] J. A. Zakarian; C. J. King, "Volatiles loss in the nozzle zone during spray drying of emulsions," Ind. Eng. Chem. Process Des. Dev., vol. 21, no. 1, pp. 107-113, Jan. 1982.https://doi.org/10.1021/i200016a019

[98] I. Zbicinski; A. Delag; C. Strumillo; J. Adamiec "Advanced experimental analysis of drying kinetics in spray drying," Chem. Eng. J., vol. 86, no. 1-2, pp. 207-216, Feb. 2002. https://doi.org/10.1016/S13858947(01)00291-1

[99] A. Tolun; Z. Altintas; N. Artik, "Microencapsulation of grape polyphenols using maltodextrin and gum arabic as two alternative coating materials: Development and characterization," J. Biotechnol., vol. 239, pp. 23-33, Dec. 2016. https://doi.org/10.1016/j.jbiotec.2016.10.001

[100] S. Y. Chong; C. W. Wong, "Effect of spray dryer inlet temperature and maltodextrin concentration on colour profile and total phenolic content of Sapodilla (Manilkara zapota) powder," Int. Food Res. J., vol. 24, no. 6, pp. 2543-2548, Dec. 2017. https://cutt.ly/qlAtput

[101] C. Saenz Hernandez; S. Tapia; J. Chavez; P. Robert, "Microencapsulation by spray drying of bioactive compounds from cactus pear (Opuntia ficus-indica)," Food Chem., vol. 114, no. 2, pp. 616-622, May. 2009. 
https://doi.org/10.1016/i.foodchem.2008.09.095

[102] V. Sharma; A. Bhardwaj, "29 Scanning electron microscopy (SEM) in food quality evaluation," in Evaluation Technologies for Food Quality, Elsevier, 2019, pp. 743-761. https://doi.org/10.1016/B978-0-12-8142172.00029-9

[103] B. Bernaola Guevara; B. Y. Quispe Florentino, "Optimización del proceso de microencapsulación de aceite de palta obtenido por CO2 supercrítico," (Tesis de pregrado), Universidad Nacional del Centro del Perú Facultad de Ingeniería en Industrias Alimentarias, 2019. URL

[104] A. Bucurescu; A. C. Blaga; B. N. Estevinho; F. Rocha, "Microencapsulation of Curcumin by a Spray-Drying Technique Using Gum Arabic as Encapsulating Agent and Release Studies," Food Bioprocess Technol., vol. 11, no. 10, pp. 1795-1806, Oct. 2018. https://doi.org/10.1007/s11947-018-2140-3

[105] P. M. Figueroa Martínez; A. M. Hurtado Benavides; M. A. Ceballos Ortíz, "Microencapsulación mediante secado por aspersión de aceite de mora (Rubus glaucus) extraido con CO2 supercrítico," Rev. Colomb. Química, vol. 45, no. 2, Nov. 2016. http://dx.doi.org/10.15446/rev.colomb.quim.v45n2.57481

[106] T. Cardoso; A. Gonçalves; B. N. Estevinho; F. Rocha, "Potential food application of resveratrol microparticles: Characterization and controlled release studies," Powder Technol., vol. 355, pp. 593-601, Oct. 2019. https://doi.org/10.1016/j.powtec.2019.07.079

[107] P. K. Binsi et al., "Structural and oxidative stabilization of spray dried fish oil microencapsulates with gum arabic and sage polyphenols: Characterization and release kinetics," Food Chem., vol. 219, pp. 158-168, Mar. 2017. https://doi.org/10.1016/j.foodchem.2016.09.126

[108] S. Jokić; N. Nastić; S. Vidović; I. Flanjak; K. Aladić; J. Vladić, "An Approach to Value Cocoa Bean ByProduct Based on Subcritical Water Extraction and Spray Drying Using Different Carriers," Sustainability, vol. 12, no. 6, Mar. 2020. https://doi.org/10.3390/su12062174

[109] G. Mercado-Mercado; L. de la R. Carrillo; A. Wall-Medrano; J. A. López Díaz; E. Álvarez-Parrilla, "Compuestos polifenólicos y capacidad antioxidante de especias típicas consumidas en México," Nutr. Hosp., vol. 28, no. 1, pp. 36-46, 2013. URL

[110] R. L. Prior; X. Wu; K. Schaich, "Standardized Methods for the Determination of Antioxidant Capacity and Phenolics in Foods and Dietary Supplements," J. Agric. Food Chem., vol. 53, no. 10, pp. 4290-4302, May. 2005. https://doi.org/10.1021/jf0502698 